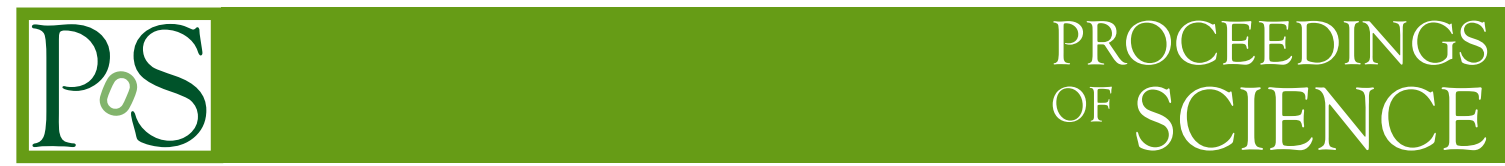

\title{
Charm mixing at Belle
}

\author{
Tao Peng ${ }^{* \dagger}$ \\ University of Science and Technology of China \\ E-mail: Dtd8606dmail.ustc.edu.cn
}

We report recent charm mixing results using $D^{0} \rightarrow K_{S}^{0} \pi^{+} \pi^{-}$and $D^{0} \rightarrow K^{+} K^{-}, \pi^{+} \pi^{-}$based on the data produced by the KEKB collider and collected with the Belle detector.

36th International Conference on High Energy Physics,

July 4-11, 2012

Melbourne, Australia

* Speaker.

$\dagger$ on behalf of the Belle collaboration. 


\section{Introduction}

In $D^{0}-\bar{D}^{0}$ mixing phenomenon, the neutral charm mesons oscillate with each other by a mixture of two mass eigenstates $\left|D_{1,2}>=p\right| D^{0}> \pm q \mid \bar{D}^{0}>$, where $|p|^{2}+|q|^{2}=1$. The physical eigenstates must have different masses $m_{1}, m_{2}$ and widths $\Gamma_{1}, \Gamma_{2}$. The mixing parameters $\mathrm{x}, \mathrm{y}$ define the masses and widths differences of the two mass eigenstates $x=\left(m_{1}-m_{2}\right) / \Gamma$ and $y=\left(\Gamma_{1}-\Gamma_{2}\right) / \Gamma$, where $\Gamma=\left(\Gamma_{1}+\Gamma_{2}\right) / 2$. The charm mixing rate is predicted to be small in standard model(SM). So measuring of mixing parameters and searching for $\mathrm{CP}$ violation is important for determining the physics beyond SM. Different $D^{0}$ decay modes show sensitivity to different combination of $\mathrm{x}$ and y. We report here two of them.

2. $D^{0} \rightarrow K_{S}^{0} \pi^{+} \pi^{-}$

In self conjugated decay $D^{0} \rightarrow K_{S}^{0} \pi^{+} \pi^{-}$, the initially produced $D^{0}$ or $\bar{D}^{0}$ 's decay amplitudes can be expressed as a function of time

$$
\begin{aligned}
& \mathscr{M}\left(m_{+}^{2}, m_{-}^{2}, t\right)=\frac{1}{2}\left(e_{1}(t)+e_{2}(t)\right) \mathscr{A}\left(m_{+}^{2}, m_{-}^{2}\right)+\frac{q}{2 p}\left(e_{1}(t)-e_{2}(t)\right) \overline{\mathscr{A}}\left(m_{+}^{2}, m_{-}^{2}\right), \\
& \overline{\mathscr{M}}\left(m_{+}^{2}, m_{-}^{2}, t\right)=\frac{1}{2}\left(e_{1}(t)+e_{2}(t)\right) \overline{\mathscr{A}}\left(m_{+}^{2}, m_{-}^{2}\right)+\frac{p}{2 q}\left(e_{1}(t)-e_{2}(t)\right) \mathscr{A}\left(m_{+}^{2}, m_{-}^{2}\right),
\end{aligned}
$$

where $\mathscr{A}$ and $\overline{\mathscr{A}}$ are the decay amplitudes for $D^{0}$ and $\bar{D}^{0}$ as a function of Dalitz-plot variables $\left(m_{+}^{2}, m_{-}^{2}\right)=\left(m_{K_{S}^{0} \pi^{+}}^{2}, m_{K_{S}^{0} \pi^{-}}^{2}\right)$, and $e_{1,2}(t)=e^{-i\left(m_{1,2}-\left(i \Gamma_{1,2} / 2\right)\right) t}$. Assuming CP is conserved, $p / q=1$ and $\overline{\mathscr{A}}\left(m_{+}^{2}, m_{-}^{2}\right)=\mathscr{A}\left(m_{-}^{2}, m_{+}^{2}\right)$. Upon squaring $\mathscr{M}$ and $\overline{\mathscr{M}}$, one can obtain the decay rates for $D^{0}$ and $\bar{D}^{0}$, where neutral mesons oscillate with each other through the terms $\cosh (y \Gamma t), \sinh (y \Gamma t)$, $\cos (x \Gamma t)$, and $\sin (x \Gamma t)$. So a time-dependent Dalitz-plot analysis of $D^{0} \rightarrow K_{S}^{0} \pi^{+} \pi^{-}$allows one to

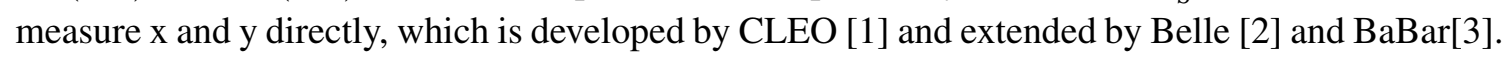

We select the $D^{0}$ and $\bar{D}^{0}$ events by decay chain $D^{*+} \rightarrow D^{0} \pi_{s}^{+}$and $D^{0} \rightarrow K_{S}^{0} \pi^{+} \pi^{-}$. The flavor of the neutral D meson is tagged by the charge of slow pion $\pi_{s}^{+}$. The final states are fully reconstructed via two kinematic variables: the invariant mass of $K_{S}^{0} \pi^{+}$and $K_{S}^{0} \pi^{-}$. We project the flight length to momentum vector and transform it to $D^{0}$ center-of-mass system to obtain the $D^{0}$ decay time t and its uncertainty $\sigma_{t}$. To suppress the combinatorial background and events from B decays, we required $D^{*+}$ momentum in the center-of-mass $(\mathrm{CM})$ frame to be greater than $2.5 \mathrm{GeV} / \mathrm{c}$ and 3.1 Gev/c for $\Upsilon(4 \mathrm{~S})$ and $\Upsilon(5 \mathrm{~S})$ data respectively. Two observables are used to determine the yield of signal and backgrounds: the invariant mass of $D^{0}$ daughters $M=m_{K_{S}^{0} \pi^{+} \pi^{-}}$and the energy released from $D^{*+}$ decay $Q=m_{K_{S}^{0} \pi^{+} \pi^{-} \pi_{s}}-m_{K_{S}^{0} \pi^{+} \pi^{-}}-m_{\pi_{s}}$.

The Dalitz distribution of $D^{0} \rightarrow K_{S}^{0} \pi^{+} \pi^{-}$are expressed as a sum of quasi-two-body amplitudes. We adopt 12 intermediate resonances for the $\mathrm{P}$ and $\mathrm{D}$ wave, $\mathrm{K}$-matrix parameterization for the $\pi \pi \mathrm{S}$-wave [䧃] and the same description as [目] for the $K_{S}^{0} \pi \mathrm{S}$ wave. We try optional models by using different combination of alternative formalism and by adding or removing resonances with small fractions. The final DP parameterization is optimized according likelihood and $\chi^{2}$ test.

To extract the mixing parameters $\mathrm{x}$ and $\mathrm{y}$, an unbinned maximum likelihood fit is performed. We parameterize the signal's PDF in a normalized form 


$$
p_{\text {sig }}\left(m_{-, i}^{2}, m_{+, i}^{2}, t_{i}\right)=\frac{\int_{0}^{+\infty} d t^{\prime} R\left(t_{i}-t^{\prime}\right)\left|\mathscr{M}\left(m_{-, i}^{2}, m_{+, i}^{2}, t^{\prime}\right)\right|^{2} \varepsilon\left(m_{-, i}^{2}, m_{+, i}^{2}\right)}{\int_{0}^{+\infty} d t \int_{D} d m_{-}^{2} d m_{+}^{2}\left|\mathscr{M}\left(m_{-}^{2}, m_{+}^{2}, t\right)\right|^{2} \varepsilon\left(m_{-}^{2}, m_{+}^{2}\right)}
$$

where i runs over all selected event candidates, $R\left(t_{i}-t^{\prime}\right)$ is the resolution function for $D^{0}$ decay time, the efficiency $\varepsilon\left(m_{-}^{2}, m_{+}^{2}\right)$ is described by a cubic polynomial with symmetry for $m_{-}^{2}$ and $m_{+}^{2}$ obtained from Monte Carlo fit.

The distribution for combinatorial background is determined in $\mathrm{M}$ sideband. The random $\pi^{+}$background contains a mixture of $D^{0}$ and $\bar{D}^{0}$ decays.The Dalitz amplitude can be written as $p_{\text {rnd }}\left(m_{-}^{2}, m_{+}^{2}\right)=\left(1-f_{\mathrm{w}}\right)\left|\mathscr{A}\left(m_{-}^{2}, m_{+}^{2}\right)\right|^{2}+f_{\mathrm{w}}\left|\mathscr{A}\left(m_{+}^{2}, m_{-}^{2}\right)\right|^{2}$, where $f_{\mathrm{w}}$ is a fraction of wrong charge tagged events in this background. We extracted $f_{\mathrm{w}}=0.511 \pm 0.003$ from $Q$ sideband. The fitting procedure is validated with fully simulated Monte Carlo experiments with background included.
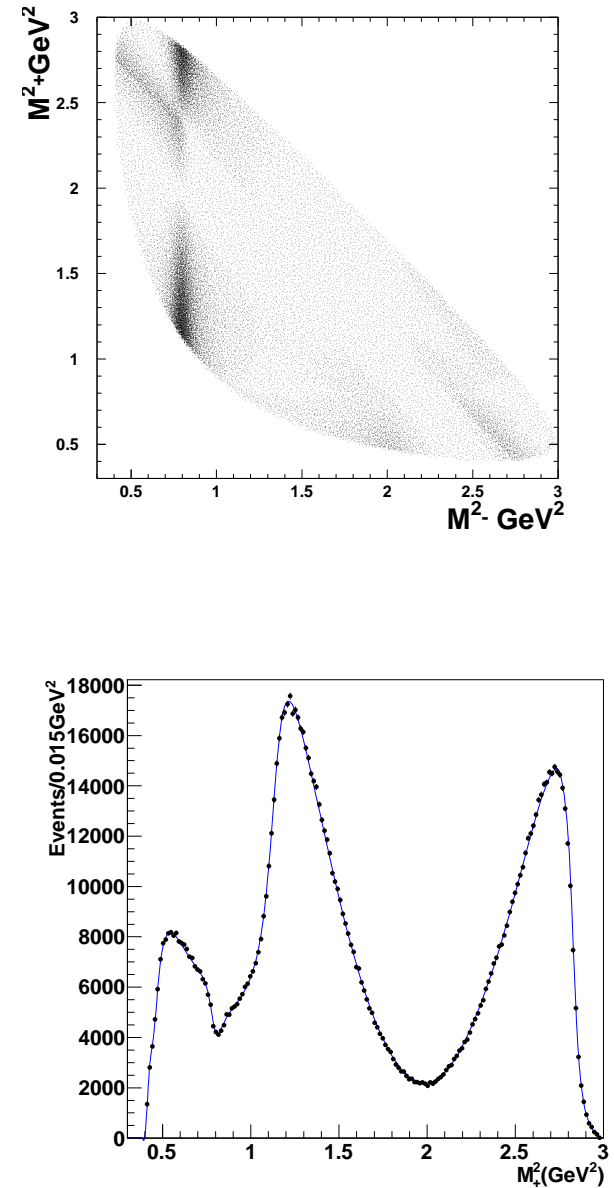

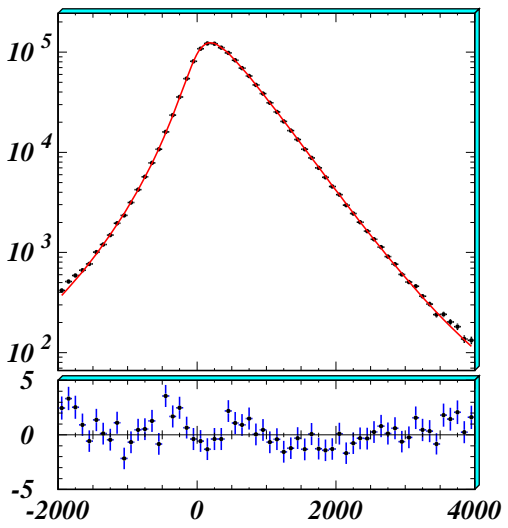

$t(f s)$

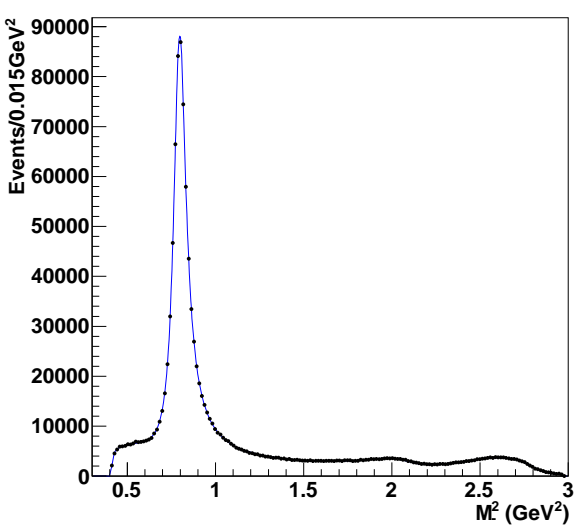

Figure 1: Dalitz-plot distribution, decay time, and $m_{-}^{2}, m_{+}^{2}$ projections for Data fit.

The fit results of data are shown in Fig.W. The fit gives $x=(0.56 \pm 0.19) \%, y=(0.30 \pm 0.15) \%$, $\tau_{D^{0}}=(410.3 \pm 0.45) f s$, the latter one consistent with world average [[5]. There are two categories 
of systematic uncertainties arising from experiment and $D^{0}$ decay model(summarized in Table ח, ). The dominant contributions for experimental and Dalitz model sources are from the the fitting performances and the parameterizations of angular dependence respectively.

Table 1: The sources of experimental systematic uncertainty. The positive and negative errors are added in quadrature separately.

\begin{tabular}{lcc}
\hline Source & $(\Delta x)\left(\times 10^{-4}\right)$ & $(\Delta y)\left(\times 10^{-4}\right)$ \\
\hline Time resolution of signal & -1.39 & -0.92 \\
Combinatorial background time's PDF & +1.74 & +1.65 \\
Errors on combinatorial time parameters & \pm 0.77 & \pm 1.57 \\
Backgrounds' Dalitz dependence on time & -4.76 & -3.55 \\
Fraction of wrong tagged events $f_{w}$ & -0.67 & -0.45 \\
Efficiency & -1.13 & -2.09 \\
Best Candidate selection & +1.05 & +1.87 \\
$K^{*}(892)$ DCS/CF reduced by 5\% & -7.28 & +2.29 \\
$K_{2}^{*}(1430)$ DCS/CF reduced by 5\% & +1.71 & -0.67 \\
Normalization of random background N & \pm 0.27 & \pm 0.13 \\
Normalization of combinatorial background & \pm 0.13 & \pm 0.24 \\
\hline Total & ${ }_{-8.94}^{+2.78}$ & +3.74 \\
\hline
\end{tabular}

Table 2: The sources of modeling systematic uncertainty. The positive and negative errors are added in quadrature separately.

\begin{tabular}{lcc}
\hline Fit model & $(\Delta x)\left(\times 10^{-4}\right)$ & $(\Delta y)\left(\times 10^{-4}\right)$ \\
\hline Form factors $F_{r}, F_{D}$ & +4.05 & +2.35 \\
Resonances' widths dependence $\Gamma\left(q^{2}\right)$ & +3.33 & -1.61 \\
Remove $K^{*}(1680)^{+}$ & -1.78 & -3.02 \\
Remove $K^{*}(1410)^{ \pm}$ & -1.16 & -3.62 \\
Remove $\rho(1450)$ & +2.13 & +0.30 \\
K-matrix formalism & -2.16 & +1.79 \\
Angular dependence & -8.46. & -3.86 \\
Resonances' M \& $\Gamma$ errors & \pm 1.40 & \pm 1.21 \\
\hline Total & ${ }_{-9.09}^{+5.83}$ & +3.21 \\
\hline
\end{tabular}

3. $D^{0} \rightarrow K^{+} K^{-}, \pi^{+} \pi^{-}$

Another evaluation method of $D^{0}-\bar{D}^{0}$ mixing is measuring the lifetime difference between $\mathrm{CP}$ even decays and its CP odd decays

$$
y_{C P}=\frac{\tau\left(D^{0} \rightarrow K^{-} \pi^{+}\right)}{\tau\left(D^{0} \rightarrow h^{+} h^{-}\right)}-1
$$

where h denotes $K$ or $\pi$. The $y_{C P}$ is equal to $\mathrm{y}$ in the absence of CP violation. 
If $\mathrm{CP}$ is violated in charm decays, the $\mathrm{CP}$ violating parameter can be defined as the lifetime asymmetry between $D^{0}$ and $\bar{D}^{0}$ to the same CP eigenstate.

$$
A_{\Gamma}=\frac{\tau\left(\bar{D}^{0} \rightarrow K^{-} K^{+}\right)-\tau\left(D^{0} \rightarrow K^{+} K^{-}\right)}{\tau\left(\bar{D}^{0} \rightarrow K^{-} K^{+}\right)+\tau\left(D^{0} \rightarrow K^{+} K^{-}\right)}
$$

To extract the proper time in different decays, we parameterized the proper decay time distribution by

$$
f(t)=\frac{N}{\tau} \int e^{-t^{\prime} / \tau} R\left(t-t^{\prime}\right) d t^{\prime}+B(t)
$$

The background distribution $B(t)$ is estimated by a fit to the sideband events. To account for the observed dependence between $D^{0}$ proper time mean value and $D^{0}$ polar angle [四], we performed the fit in each bins of $\cos \theta^{*}$, where $\theta^{*}$ is the $D^{0}$ polar angle in CMS.

Fig. $\square$ shows the results of the fits in bins of $\cos \theta^{*}$ for $y_{C P}, A_{\Gamma}$ and $D^{0}$ lifetime $\tau$. We obtained the average $y_{C P}=(1.11 \pm 0.22 \pm 0.11) \%, A_{\Gamma}=(-0.03 \pm 0.20 \pm 0.08) \%$, and $\tau_{D^{0}}=(408.46 \pm$ $0.54) f s$ by a least square fit to a constant. The measured $D^{0}$ lifetime $\tau$ is consistent with the world average [回].
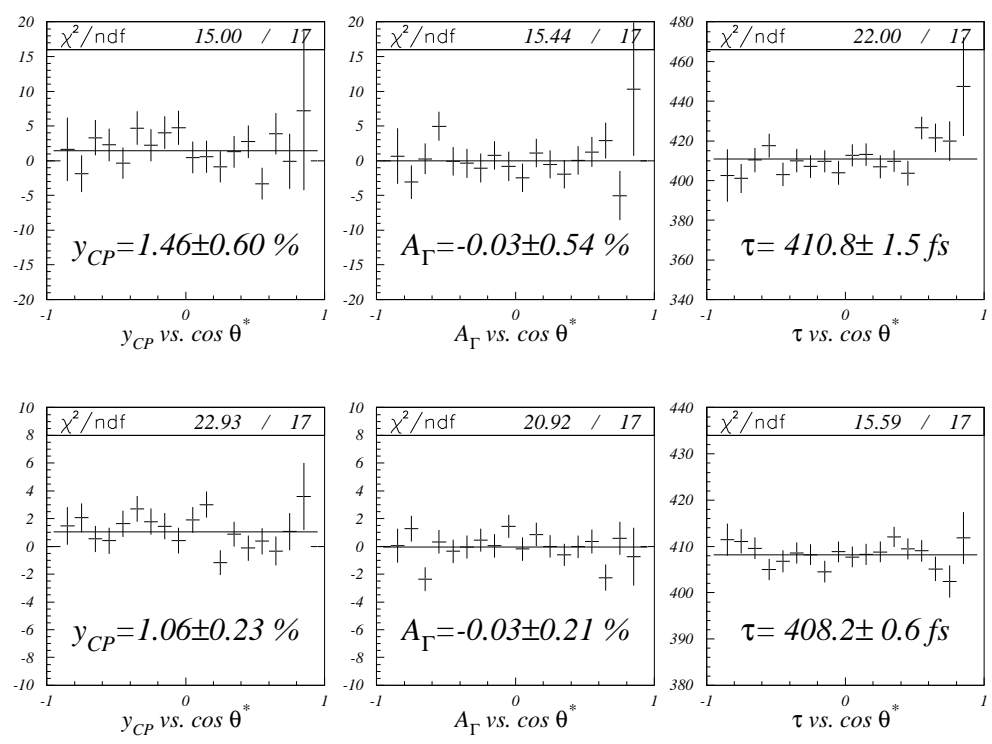

Figure 2: Results of $y_{C P}, A_{\Gamma}$ and $\tau_{D^{0}}$ in simultaneous fits in bins of $\cos \theta^{*}$ (points with error bars. Top(bottom) plots are for data collected by 3-layer(4-layer) silicon detector respectively.

\section{Summary}

In summary, using time-dependent Dalitz plot analysis of $D^{0} \rightarrow K_{S}^{0} \pi^{+} \pi^{-}$, we measure

$$
\begin{aligned}
& x=\left(0.56 \pm 0.19_{-0.09-0.09}^{+0.03+0.06}\right) \%, \\
& y=\left(0.30 \pm 0.15_{-0.05-0.06}^{+0.04+0.03}\right) \%,
\end{aligned}
$$


which is most precise to date and consistent with previous measurements [] []]].

In $D^{0} \rightarrow K^{+} K^{-}, \pi^{+} \pi^{-}$, we obtain

$$
\begin{gathered}
y_{C P}=(1.11 \pm 0.22 \pm 0.11) \%, \\
A_{\Gamma}=(-0.03 \pm 0.20 \pm 0.08) \% .
\end{gathered}
$$

The significance of $y_{C P} \neq 0$ confirmed evidence for $D^{0}-\bar{D}^{0}$ mixing, and the result for $A_{\Gamma}$ is consistent with no CP violation.

\section{References}

[1] D. M. Asner et al.(CLEO Collaboration), Phys. Rev.D 72, 012001 (2005).

[2] L. M. Zhang et al.(Belle Collaboration), Phys.Rev.Lett. 99,131803(2007)

[3] B. Aubert et al.(BaBar Collaboration), Phys.Rev.Lett.105, 081803 (2010)

[4] B. Aubert et al.(BaBar Collaboration), Phys. Rev. D 78, 034023 (2008).

[5] K. Nakamura et al.(Particle Data Group), J. Phys. G: Nucl. Part. Phys. 37, 075021 (2010).

[6] M. Starič et al.(Belle Collaboration), Phys. Rev. Lett. 37, 211803 (2007) 